\title{
Barriers to Mobility During Hospitalization from the Perspectives of Older Patients and Their Nurses and Physicians
}

Cynthia J. Brown, MD, MSPH ${ }^{1,2,3}$

Beverly R. Williams, $\mathrm{PhD}^{1,2,3}$

Lesa L. Woodby, PhD ${ }^{1,2,4}$

Linda L. Davis, PhD, RN ${ }^{5,6}$

Richard M. Allman, MD ${ }^{1,2,3}$

${ }^{1}$ Birmingham/Atlanta Veterans Affairs Geriatric Research, Education, and Clinical Center (GRECC), Birmingham, Alabama

${ }^{2}$ Department of Medicine, Division of Gerontology, Geriatrics, and Palliative Care, University of Alabama at Birmingham, Birmingham, Alabama

${ }^{3}$ Center for Aging, University of Alabama at Birmingham, Birmingham, Alabama

${ }^{4}$ Department of Family and Community Medicine, University of Alabama at Birmingham, Birmingham, Alabama

${ }^{5}$ School of Nursing, Duke University, Durham, North Carolina

${ }^{6}$ Center for the Study of Aging and Human Development, Duke University, Durham, North Carolina

The authors are indebted to the patients, nurses, and resident physicians at University Hospital who participated in the study; to Stephanie Stone, MBA, for her invaluable assistance with study execution; and to Robert H. Brown, MDiv, for his critical review of the manuscript.

This work was presented in part at the annual meeting of the Gerontological Society of America in Orlando, Florida, in November 2005.
BACKGROUND: Low mobility is common during hospitalization and is associated with adverse outcomes. Understanding barriers to the maintenance or improvement of mobility is important to the development of successful interventions.

OBJECTIVES: To identify barriers to mobility during hospitalization from the perspectives of older patients and their primary nurses and physicians, to compare and contrast the perceived barriers among these groups, and to make a conceptual model.

DESIGN: Qualitative interviews analyzed and interpreted using a grounded theory approach.

SETTING: Medical wards of a university hospital.

PARTICIPANTS: Twenty-nine participants-10 patients $\geq 75$ years, 10 nurses, and 9 resident physicians.

MEASUREMENTS: Participants were interviewed using a semistructured interview guide, with similar questions for patients and health care providers. Interviews were audiotaped, transcribed, and reviewed for common themes by independent reviewers. Perceived barriers to mobility were identified, and their nature and frequency were examined for each respondent group.

RESULTS: Content analysis identified 31 perceived barriers to increased mobility during hospitalization. Barriers most frequently described by all 3 groups were: having symptoms (97\%), especially weakness (59\%), pain (55\%), and fatigue (34\%); having an intravenous line $(69 \%)$ or urinary catheter (59\%); and being concerned about falls (79\%). Lack of staff to assist with out-of-bed activity was mentioned by patients $(20 \%)$, nurses $(70 \%)$, and physicians $(67 \%)$. Unlike patients, health care providers attributed low mobility among hospitalized older adults to lack of patient motivation and lack of ambulatory devices.

CONCLUSIONS: Recognizing and understanding perceived barriers to mobility during hospitalization of older patients is an important first step toward developing successful interventions to minimize low mobility. Journal of Hospital Medicine 2007;2:305-313. () 2007 Society of Hospital Medicine.

KEYWORDS: mobility limitation, accidental falls, geriatrics, hospital care, qualitative methods.

$\mathbf{T}$ he adverse outcomes associated with hospitalization of older patients, such as functional decline and increased nursing home placement, have been well documented. ${ }^{1-9}$ Low mobility, defined as being limited to a bed or chair, has also been associated with these adverse outcomes, even after controlling for severity of illness. ${ }^{10}$ Early ambulation has been a common practice for years following many types of orthopedic operations, including hip fracture repair and total joint replace- 


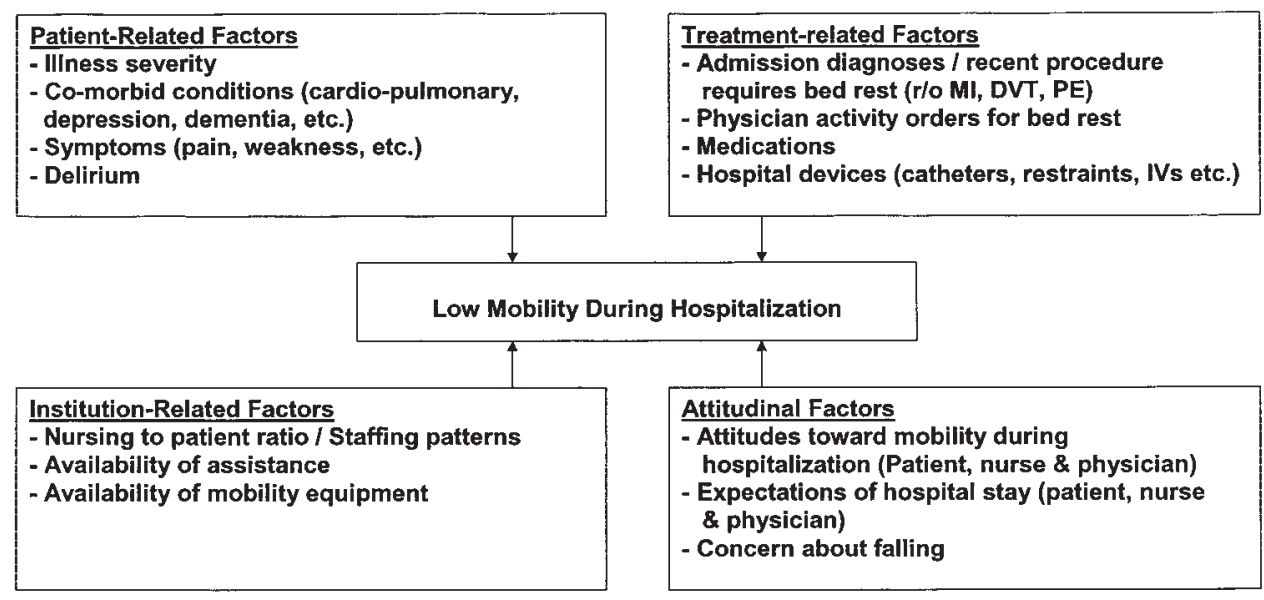

FIGURE 1. Original conceptual model of barriers to mobility during hospitalization of older persons ( ${ }^{*} \mathrm{MI}$, myocardial infarction; DVT, deep-vein thrombosis; PE, pulmonary embolus; IV, intravenous line).

ment. ${ }^{11,12}$ A recent study demonstrated that time to ambulation after surgery was an independent predictor of the development of postoperative complications such as pneumonia and delirium. ${ }^{13}$

In the early 1980s, early ambulation became the cornerstone of cardiac rehabilitation after acute myocardial infarction. ${ }^{14,15}$ Until recently, with the exception of post-myocardial infarction, the use of early ambulation for patients admitted with medical illnesses has not been studied. In the last few years, researchers have begun to explore the use of early ambulation for patients after cardiac catheterization and for those admitted with deep-vein thrombosis and pneumonia. ${ }^{16-20}$ Although many of these studies have been small, they have found early ambulation not to be associated with worse outcomes. Indeed, a study of early ambulation for patients with community-acquired pneumonia demonstrated decreased hospital costs and increased functional ability prior to discharge. ${ }^{20}$

Although the literature documents the adverse consequences associated with bed rest $\mathrm{t}^{21-23}$ and the beneficial effects of early ambulation, patients continue to spend a significant amount of their hospital stay limited to a bed or a chair. The prevalence of low mobility in older patients ranges from $23 \%$ to $33 \%$ during hospitalization for medical illness. ${ }^{9,10}$ Despite the high prevalence and the associated adverse outcomes of low mobility among hospitalized older adults, the factors associated with low mobility in the hospital setting have not been systematically explored. Identification of such factors is the first step toward recognizing potentially modifiable factors and developing targeted interventions to improve hospital care.

We conceptualized a variety of factors or barriers that could potentially affect the level of mobility achieved by older hospitalized patients. Using professional experience and expert opinion, a conceptual model of potential barriers to mobility was developed (Fig. 1). As Figure 1 illustrates, the model has 4 major categories: patient-related factors, including illness severity or comorbid conditions; treatment-related factors such as catheters and intravenous lines; institution-related factors such as nursing-to-patient ratio; and attitudinal factors related to perspectives on mobility and concerns about falling. This model was reviewed and feedback provided by a multidisciplinary group of colleagues including physicians, nurses, physical therapists, medical educators, and medical sociologists.

The objectives of this study were to employ qualitative methodology to identify and contextualize perceived barriers to mobility during hospitalization from the perspectives of older patients, their primary nurses, and their resident physicians; to compare and contrast the perceived barriers among these 3 groups; and to compare perceived barriers to mobility with our conceptual model.

\section{METHODS}

\section{Setting and Patients}

Patients aged $\geq 75$ years admitted to the medical wards of the University Hospital either directly or through the Emergency Department were recruited for this study. In addition, the primary nurses and 
resident physicians of enrolled patients were also recruited because of their roles in providing handson care to these patients in the university hospital setting. This project was supported in part by a VA Research Career Development Award and a training support grant from the Hartford Foundationfunded Southeast Center of Excellence in Geriatric Medicine. Written informed consent for participation was obtained from the patients, their nurses, and their resident physicians according to procedures approved by the Institutional Review Board of the University of Alabama at Birmingham. Recruitment was continued until no new barriers to hospital mobility were identified and data saturation was achieved. In all, 29 persons were enrolled: 10 patients, 10 nurses, and 9 physicians.

Patient exclusion criteria included factors that made it difficult for an individual to participate in the interview and to self-report on mobility: (1) inability to be interviewed (ie, obtunded, aphasic), (2) a significant language barrier, requiring a translator, (3) Mini Mental State Examination Score ${ }^{24}<$ 16, (4) delirium at the time of the interview as documented by the Confusion Assessment Method $(\mathrm{CAM})^{25}$, and (5) self-reported inability to ambulate or transfer 2 weeks prior to hospital admission; Additional exclusion criteria were (6) previous enrollment in the study by the patient, the primary nurse, or the physician; and (7) refusal by patient, family, or physician to participate.

\section{Questionnaire Development}

A semistructured interview guide approach was used to encourage participants to discuss their perception of barriers to mobility during hospitalization. Pilot testing at a local retirement center was used to revise the initial patient questionnaire. Domains of inquiry were identified through the pilot testing and based on the conceptualized model (Fig. 1). These domains included attitudes toward mobility, expectations of care regarding walking/ mobility, patient-related factors that influence mobility, situational factors that influence mobility, and specific perceived barriers to mobility. Similar but not identical questions were used for patients and health care providers so answers could be compared between participant subgroups. For example, patients were asked, "What might make it easier for you to get out of bed and walk more frequently or for longer periods than you are now?" and health care providers were asked, "What would make it easier for the patient to get out of bed and walk more frequently or for longer periods of time?" Responses were categorized as being spontaneous or prompted depending on how the responses were elicited. Spontaneous responses about mobility barriers were elicited during general questioning about the hospital stay; for example: "Tell me what you think about getting out of bed and moving around during this hospitalization?" Prompted responses were elicited by asking a specific question about a potential barrier: "Do you have any concerns about falling during your hospital stay?"

\section{Data Analysis}

Interviews were conducted by the principal investigator (PI) between September 2004 and January 2005. Using an iterative approach, analysis began after the first interview with emerging themes being explored in subsequent interviews. All interviews were audiotaped and transcribed verbatim by a medical transcriptionist. Each participant was assigned a unique identifier. Interviews were reviewed by the PI to verify content. Participants were also given an opportunity to review the content of their interviews, with $76 \%$ choosing to do this. Using the grounded theory approach, an analytic technique that systematically analyzes raw interview data to generate hypotheses and develop theory; the data were analyzed by generating categories and themes. ${ }^{26}$

Interviews were independently reviewed and coded by the research assistant and the PI, with a third reviewer available to resolve disputes throughout the analyses. Initially, reviewers coded any item they believed to fit the category of barriers. Discrepancies between reviewers led to review of the original data to determine if the item could be considered a barrier. This process continued until the reviewers were in agreement about what to include as a barrier. Next, the themes generated by the coders were compared. Any discrepancies again led to review of the original data and revision of themes as indicated. This process continued until agreement was achieved for categories and themes between the coders for each of the 29 interviews. Independent themes were identified and counted and are presented as percentages for the purpose of comparing and contrasting between groups.

\section{RESULTS}

Fifty-seven patients age $\geq 75$ years were admitted to the medical service during the study period. Of those, 18 were excluded because they were too ill 
TABLE 1

Baseline Characteristics of Study Patients

\begin{tabular}{ll}
\hline Characteristic & Mean $( \pm$ SD) or N (\%) \\
\hline Age (years) & $84( \pm 6.0)$ \\
Sex - Male & $3(30)$ \\
$\quad$ - Female & $7(70)$ \\
Race - Black & $3(30)$ \\
- White & $7(70)$ \\
Mini Mental State Examination score & $24( \pm 3.3)$ \\
Number of comorbidities & $6( \pm 2.3)$ \\
Number of medications at admission & $9( \pm 2.3)$ \\
\hline
\end{tabular}

according to their physician $(\mathrm{n}=6)$, had a nonmedical illness $(n=5)$, were discharged before being interviewed $(n=3)$, or were being cared for by a previously enrolled nurse or physician $(n=4)$. Of the 39 who were eligible for the study, 7 declined participation (6 patients, 1 physician), and data were lost for 3 as a result of technical difficulties with the tape recorder. A total of 29 participants had an interview transcribed: 10 patients, 10 nurses, and 9 physicians. As in other qualitative inquiries, data saturation was achieved after about 10 interviews, ${ }^{27}$ but we continued recruitment to permit comparison of themes identified by patients, nurses, and physicians.

Table 1 presents baseline characteristics of the patients. The mean age of the nurses was $34.9 \pm 9.9$ years, all were female, and $60 \%$ were black. The mean age of the resident physicians was $29.1 \pm 2.2$ years; $44 \%$ were female; and $78 \%$ were white, $22 \%$ were Latino, and none were black.

Figure 2 presents barriers most frequently noted by participants. These barriers were similar among the 3 groups for symptoms and hospitalrelated factors like catheters and lack of staff. Lack of patient motivation, lack of ambulatory devices, and medical reasons necessitating bed rest were reported more frequently by health care providers than by patients. On admission, $40 \%$ of patients had bed rest ordered. By hospital day 3, all patients had out-of-bed orders, and $70 \%$ had had physical therapy ordered.

\section{Symptoms}

Symptoms were spontaneously mentioned by $97 \%$ of participants (28 of 29) as a cause of limited mobility, with weakness cited most frequently: "I know it is going to be painful (to walk). My head swims. This side of my-all up and down to my toes is weak. When that comes, you kind of be scared." Pain and fatigue were also mentioned by more than $35 \%$ of all participants. Although $42 \%$ of health care providers (8 of 19) identified dyspnea or shortness of breath as a barrier, no patients reported this symptom as a barrier.

\section{Medical Devices}

When asked directly about the impact of urinary catheters and intravenous lines, most patients expressed the belief that the device could either be disconnected or carried and therefore was not a barrier: "They can take that off [the catheter] and hook it on the wall." Only $30 \%$ of the patients described their mobility as hampered by the medical devices, and only 1 patient (10\%) spontaneously indicated the catheter was a barrier: "I have had that catheter hooked up to me until today. That was a relief to get that out...I couldn't hardly do nothing with that."

In contrast, most health care providers (89\%) believed medical devices adversely affected mobility. However, only $32 \%$ spontaneously noted the IV or catheter was a barrier, with the other $67 \%$ requiring prompting to consider the medical devices as barriers. The rationale for this belief varied widely. Some providers focused on the impact of the patient having to push the IV pole: "They get tangled, they hurt when they are mobilized. You have to push the little cart, so patients actually need some assistance." Other providers mentioned the impact of a patient's medical devices on the nurse's ability to assist patients out of bed: "I hate to say it, but I think on some days, it does [affect mobility]. You have patients who have TPN and blood and Foleys and chest tubes, you are probably less likely to [get people out of bed]." One physician commented on a less obvious impact of catheters: "Some people might be embarrassed to walk around with that Foley catheter."

\section{Need for Assistance and Lack of Staff}

Patients, nurses, and physicians alike spontaneously identified the patient's need for assistance with ambulation as a barrier. This observation was frequently followed by comments about staff shortages and time constraints that limited the availability of staff to assist patients with ambulation. The impact of a perceived lack of staff was expressed differently by patients and health care providers. Patients frequently talked about not wanting to bother the nurses: "I know it would be good for me, but I just don't want to impose upon them"; "I try 


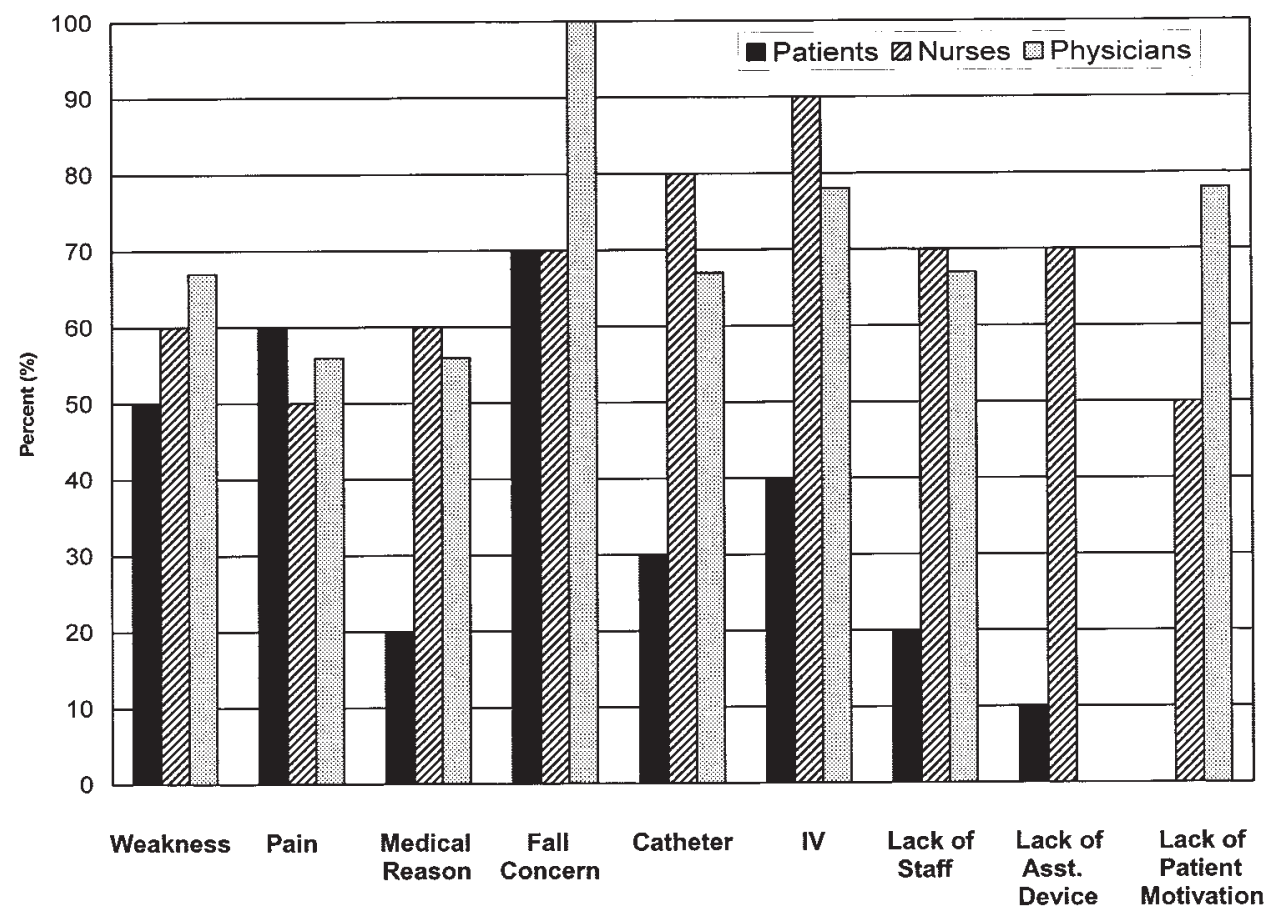

FIGURE 2. Barriers frequently noted by participants, subdivided into patients, nurses, and physicians For each barrier reported, the dark bar corresponds to patient responses, the striped bar to nurse responses, and the dotted bar to physician responses.

not to worry the girls to take me walking because they have their own patients." Health care providers focused on the variety of other nursing duties that tended to take precedence over helping patients with mobility: "I just don't think the nurses have time. They are too busy doing other things to walk their patients up and down the hallway. I think if you really want your patient up walking down the hall, you need to have a relative help them or physical therapy." Among staff, the most frequently mentioned solution was to refer patients to physical therapy, a strategy endorsed equally by nurses and physicians: "That is why we try to encourage the doctors to order physical therapy, because we don't have time to ambulate patients in the hallway like the doctor expects."

\section{Lack of Ambulatory Devices}

The nurses frequently mentioned the absence of ambulatory devices to assist patients with walking. Nurses also expressed concern about the ability of patients to walk safely without such devices: "Sometimes if a patient requires a lot of help, then I think you really need to involve physical therapy to ensure the safety of the person getting up. Because a lot of times you are limited with equipment that you need and they [physical therapists] have that equipment to make sure they don't fall." No physicians and only 1 patient cited lack of ambulatory devices as a barrier to mobility.

\section{Fear of a Patient Falling}

The potential for a fall during a hospital stay was of concern for more than $75 \%$ of the participants, with physicians spontaneously expressing concern for falls as a perceived barrier more frequently than did patients or nurses: "I probably don't encourage her to move as much as I should. And when I do, I tell her to be sure to have someone with her when she is getting up. I am probably a little more protective because I am afraid she might fall." Another physician stated, "I think nurses in general would prefer the patient to stay in bed. I believe they perceive it as a risk for falls and a risk for pulling out their IVs or any other medical device, and it is probably not viewed as an important factor in someone who is recovering from an illness." Another physician noted fall prevention was a secondary reason for limited mobility but also expressed the idea that bed rest was easier than mobility: "While they are in 
bed they are not giving trouble to anybody. It is less work and, second, because of liability issues in terms of patients falling and hurting themselves while they are in the hospital. I think everybody is very concerned with that, but I think mainly because it is less work." Although $68 \%$ of health care providers spontaneously noted falls as a barrier to mobility, only 1 patient spontaneously verbalized this concern. However, with prompting to consider falls a barrier, $60 \%$ of patients stated falls were a potential barrier: "As old as I am, your legs don't last long and they give away. It would be dangerous because I haven't got the strength. Now, since I've fallen, yes, I have to be careful."

\section{Lack of Patient Motivation}

Lack of patient motivation was mentioned by $50 \%$ of the nurses and $78 \%$ of the physicians and was often linked to a patient's age: "I just think he is older now, and he is not as motivated as younger people are, and he has been through so much. I just don't think he wants to do it." "I don't know if it is because it is the elderly or because they just seem more stubborn. They are sometimes more content to stay in the bed, and you have to really stress to them to get up." However, none of the patients indicated a lack of motivation was the reason for not getting out of bed. Indeed, they commented on the staff's apparent lack of interest or their perception that the staff did not consider increased mobility important: "I don't believe they are going to get me out of bed while I am here. If I said I really needed to get out of bed, they try to do what you want them to do. But evidently they don't think it is that important."

\section{Hospital Environment}

Although not frequently mentioned, issues of an environment not conducive to mobility did emerge as a theme. Several patients expressed this environmental barrier as a difficulty with the hospital gowns that are required garb during a hospital stay. One patient wrote the following additional thoughts after reviewing her transcript: "Gowns lead to embarrassing moments, are designed for benefit of staff, not patients." This sentiment was echoed by a physician who, when queried about the impact of gowns, responded, "I think the gown exposes the patient $a$ lot and they might feel embarrassed to go around. And outside the hospital room, nobody wants to be perceived as sick and draw attention." Finally, 2 physicians commented on the lack of chairs in the room and the physical setup of the room not encouraging mobility: "I think that patients, when they are in the hospital, they feel they are supposed to be in bed. And they are more comfortable there and a lot of times they can see the TV better."

\section{DISCUSSION}

Many of the barriers described in the original conceptual model (Fig. 1) were cited by participants from all 3 groups: patients, nurses and physicians. These included patient-related factors like symptoms and need for assistance, concern about falls, and lack of staff to assist with ambulation. Although attitudes toward mobility were cited in the model and by participants, there was significant disagreement between the 3 groups about the cause of the attitudinal barrier. Health care providers cited lack of patient motivation, whereas patients perceived health care providers as not being interested in mobility or viewing it as important. Health care providers frequently employed stereotypes to describe the potential reasons for the perceived attitudes toward mobility, often linking lack of motivation or interest in getting out of bed to patients being old. Patients linked the lack of importance attached to mobility to the numerous duties of staff members and believed that assistance with mobility was less important than other duties. Physicians and nurses were both more likely than patients to mention factors like urinary catheters, intravenous lines, and other medical reasons that necessitated bed rest. Although more than half the nurses commented on the lack of ambulatory devices for ambulation, no physicians and only 1 patient perceived this lack to be a barrier.

The model presented appears to have face validity, with participants citing many of the factors originally identified as barriers to mobility. The original model did not include consideration of environmental factors such as the number of chairs in the room or the location of the television. Such environmental factors can be conceptualized as institution-related factors.

In addition, the impact of physician activity orders for bed rest was not specifically discussed by participants, although $45 \%$ of participants did comment on the need for bed rest because of a medical condition. A review of the medical records for activity orders indicated $40 \%$ of the patients initially had orders for bed rest. Another recent study demonstrated $33 \%$ of older patients were on bed rest at some point during their hospital stay ${ }^{10}$ and should 
be retained in the model as a treatment-related consideration.

Several barriers noted in the original model and by participants may not be modifiable, such as comorbid conditions and illness severity. But other perceived barriers may be, and recognition of these factors present potential targets for a future multicomponent intervention to enhance hospital mobility. This multicomponent approach has been highly successful for geriatric syndromes like falls ${ }^{28}$ and delirium. ${ }^{29}$

During hospitalization, a focus on early removal of catheters and intravenous lines may encourage mobility. In a recent study absence of a urinary catheter was a predictor of patients with activity limitations regaining ambulatory ability while hospitalized. ${ }^{30}$ Availability of ambulatory devices may allow nurses to ambulate patients without consulting physical therapy. Another potential solution would be a hospitalwide walking program. The feasibility of a walking program was demonstrated in a small pilot study at a community-based hospital using specially trained transporters to walk ward patients during slow periods. These periods included nights and weekends, when patients were more likely to be available and when physical therapy was often not present. On average, participants spent 2.4 days in the program, with an average of 5.6 walks per patient. However, additional research is needed, as the study was too small to demonstrate the effects of the walking program on length of stay or functional decline. ${ }^{31}$

Other barriers, while potentially modifiable, may be more difficult to address and may involve changing the culture of the hospital. For instance, concern about falls was a common theme, echoed by all 3 groups. Physicians were the most likely to spontaneously mention falls as a concern. The nurses shared this concern, frequently citing the use of bed rails as a part of their fall protocol, despite available literature demonstrating that this approach was not efficacious. ${ }^{32-34}$ Nurses also consistently reported asking patients to call for help to ambulate, yet both patients and nurses noted a lack of nursing time to assist with ambulation. The default solution providers reported using was utilizing physical therapists, who were available to walk with patients only once or twice a day. Research on the best methods to prevent falls during hospitalization is limited. Given the current medicolegal environment and the emphasis on fall prevention by the Joint Commission on Accreditation of Healthcare
Organizations and other government entities, it is not surprising that bed rest and mobility limitation are being used as a method of minimizing falls. Until data about successful fall-prevention strategies are available, minimization of mobility may remain the default solution.

Strengths of the present study include the use of qualitative methods to explore potential barriers to mobility, a method that allows participants to describe in their own words their attitudes, beliefs, and expectations about mobility during hospitalization. Face-to-face interviewing of the 3 major groups involved in the hospital experience (patients, nurses, and physicians) facilitated the collection of detailed contextualized information on factors expected to affect a patient's level of mobility during hospitalization. This enabled hypothesis generation and sensitization to issues that need further quantitative investigation with larger groups.

The study also had several limitations. First, only resident physicians were included, and their answers may not reflect the opinions of other, more experienced physicians. However, resident physicians were chosen for this study because they play important roles in delivering hands-on care in teaching hospitals and would need to be involved in any future interventions designed to enhanced patient mobility. Second, a sample size of 29 participants, approximately 10 persons in each group, may not reflect the thinking of those throughout the hospital. However, sampling was continued until no new themes or barriers emerged, and major themes emerged consistently throughout the interviews with all 3 groups. Interviews were conducted at a large, urban university hospital, and so results may not be generalizable to smaller community hospitals. Last, although the 3 major groups-patients, nurses, and physicians-were included in the interviews, the opinions of other stakeholders who may have had perspectives on mobility such as family members were not solicited and may need to be incorporated into the model.

This study presents the perceived barriers to mobility from the perspectives of patients their nurses and physicians. The modifiable and nonmodifiable factors that might affect mobility during hospitalization that made up the original theoretical model were consistent with the barriers cited by the participants. Importantly, this research has led to the identification of other barriers such as environmental factors that may also influence the mo- 
bility of older patients. This research has provided insights into potentially modifiable factors of the well-documented phenomenon of low mobility of hospitalized older persons ${ }^{10}$ and identified several targets for a multicomponent intervention to minimize low mobility. Possible interventions include a progressive walking program initiated early in the hospital stay, provision of assistive devices to patients who need them, and early removal of catheters and intravenous lines. Further research is needed to explore other factors associated with low mobility, such as specific medical conditions for which bed rest may be ordered, and to evaluate the impact that specific interventions may have on the mobility of older persons during hospitalization.

Address for correspondence and reprint requests: Cynthia J. Brown, MD, MSPH, VAMC GRECC (11-G), Room 8226, 1530 3rd Avenue South, Birmingham, AL 35294; Fax: (205) 558-4749; E-mail: cbrown@aging.uab.edu

Received 29 January 2007; revision received 5 March 2007; accepted 8 March 2007.

\section{APPENDIX}

\section{Questionnaire Guide for Patients} Attitudes toward Mobility

1. Tell me how much walking do you do at home when you are not sick.

2. Do you leave your bedroom when you are at home? Do you need help to do this?

3. Do you go out of the house when you are at home? Do you need help to do this?

4. Do you go out of your neighborhood when you are at home? Do you need help to do this?

5. Tell me what you think about getting out of bed and moving around during this hospitalization? Do you think it is a good idea or a bad one?

6 . How important do you believe it is for you to rest while you are in the hospital? Why did you choose that answer?

7. How important do you believe it is for you to walk while you are in the hospital? Why did you choose that answer?

8. Do you believe it to be dangerous for you to get up out of bed and walk? Why or why not?

9. Do you think your doctor wants you to get out of bed and walk? Why or why not?

10. Do you think your nurse wants you to get out of bed and walk? Why or why not?

\section{Expectations of Care about Walking/Mobility}

1. Can you tell me what you believe the nurse is supposed to do for you while you are in the hospital?
2. When you leave the hospital, do you believe you will be able to walk on your own, or will you need help?

Needs help: Why do you believe you will need help? What type of help will you need?

No help needed: Why do you believe you will not need help?

3. When you leave the hospital, do you believe you will be able to care for yourself, or will you need help? Needs help: Why do you believe you will need help? What type of help will you need?

No help needed: Why do you believe you will not need help?

4. If you needed help, who would be available to help you when you go home?

\section{Person Factors That Influence Mobility}

1. Tell me about the illness that brought you into the hospital.

2. How serious do you believe your illness to be? Why do you believe that?

3. How easy will it be for you to get better from this illness? Why do you believe that?

\section{Situational Factors That Influence Mobility}

1. Do you have any thoughts about what might make it easier for you to get out of bed and walk more frequently or for longer periods than you are now?

2. If you decided to go to the bathroom, would you call for help?

3. Why did you choose that answer?

\section{Perceived Barriers}

1. Tell me what, if anything, would prevent you from getting out of bed and walking during your hospital stay?

2. Do you have any concerns about falling during your hospital stay?

3. Are there other factors that influence if you will walk during your hospitalization that I haven't asked about?

\section{New Questions}

As this is a qualitative study, if participants bring up new topics that have not been previously explored with the questionnaire guide, these questions will be added to the questionnaire. Future participants will be asked the new questions. 


\section{REFERENCES}

1. McCusker J, Kakuma R, Abrahamowicz M. Predictors of functional decline in hospitalized elderly patients: a systematic review. J Gerontol Med Sci. 2002;57A:M569-M577.

2. Covinsky KE, Palmer RM, Fortinsky RH, et al. Loss of independence in activities of daily living in older adults hospitalized with medical illnesses: increased vulnerability with age. J Am Geriatr Soc. 2003; 51:451-458.

3. Sager MA, Franke T, Inouye SK, et al. Functional outcomes of acute medical illness and hospitalization in older persons. Arch Intern Med. 1996;156:645-652.

4. McVey LJ, Becker PM, Saltz CC, Feussner JR, Cohen HJ. Effect of a geriatric consultation team on functional status of elderly hospitalized patients. Ann Intern Med. 1989;110:7984.

5. Warsaw G, Moore J, Friedman S, et al. Functional disability in the hospitalized elderly. JAMA. 1982;248:847-850.

6. Narain P, Rubenstein LZ, Wieland GD, et al. Predictors of immediate and 6-month outcomes in hospitalized elderly patients. J Am Geriatr Soc. 1988;36:775-783.

7. Gillick MR, Serrell NA, Gillick LS. Adverse consequences of hospitalization in the elderly. Soc Sci Med. 1982;16:10331038.

8. Rosin AJ, Boyd RV. Complications of illness in geriatric patients in hospital. J Chronic Dis. 1966;19:307-313.

9. Lazarus BA, Murphy JB, Coletta EM, McQuade WH, Culpepper L. The provision of physical activity to hospitalized elderly patients. Arch Intern Med. 1991;151:2452-2456.

10. Brown CJ, Friedkin RJ, Inouye SK. Prevalence and outcomes of low mobility in hospitalized older patients. J Am Geriatr Soc. 2004;52:1263-1270.

11. Cameron ID, Lyle DM, Quine S. Cost effectiveness of accelerated rehabilitation after proximal femoral fracture. J Clin Epidemiol. 1994;47:1307-1313.

12. Munin MC, Rudy TE, Glynn NW, Crossett LS, Rubash HE. Early inpatient rehabilitation after elective hip and knee arthroplasty. JAMA. 1998;279:847-852.

13. Kamel HK, Iqbal MA, Mogallapu R, Maas D, Hoffman RG. Time to ambulation after hip fracture surgery: relation to hospitalization outcomes. J Gerontol. 2003;58A:1042-1045.

14. Harpur JE, Conner WT, Hamilton M, et al. Controlled trial of early mobilization and discharge from hospital in uncomplicated myocardial infarction. Lancet. 1971;2:1359-1360.

15. Abraham AS, Sever Y, Weinstein M, Dollberg M, Menczel J. Value of early ambulation in patients with and without complications after acute myocardial infarction. $N$ Engl J Med. 1975;292:719-722.

16. Allen C, Glasziou P, DelMar C. Bed rest: a potentially harmful treatment needing more careful evaluation. Lancet. 1999; 354:1229-1233.
17. Kern MJ, Cohen M, Talley JD, et al. Early ambulation after 5 French diagnostic cardiac catheterization: results of a multicenter trial. J Am Coll Cardiol. 1990;15:1475-1483.

18. Pollard SD, Munks K, Wales C, et al. Position and mobilisation post-angiography study (PAMPAS): a comparison of 4.5 hours and 2.5 hours bed rest. Hear. 2003;89:447-448.

19. Trujillo-Santos J, Perea-Milla E, Jimenez-Puente A, et al. Bed rest or ambulation in the initial treatment of patients with acute deep vein thrombosis or pulmonary embolism: findings from the RIETE Registry. Chest. 2005;127:1631-1636.

20. Mundy LM, Leet TL, Darst K, Schnitzler MA, Dunagan WC. Early mobilization of patients hospitalized with communityacquired pneumonia. Chest. 2003;124:883-889.

21. Creditor MC. Hazards of hospitalization of the elderly. Ann Intern Med. 1993;118:219-223.

22. Harper CM, Lyles YM. Physiology and complications of bedrest. J Am Geriatr Soc. 1988;36:1047-1054.

23. Hoenig HM, Rubenstein LZ. Hospital-associated deconditioning and dysfunction. J Am Geriatr Soc. 1991;39:220-222.

24. Folstein MF, Folstein SE, McHugh PR. "Mini-mental state." A practical method for grading the cognitive status of patients for the clinician. J Psychiatr Rev. 1975;12:189-198.

25. Inouye SK, van Dyck $\mathrm{CH}$, Alessi CA, Balkin S, Siegal AP, Horwitz RI. Clarifying confusion: the confusion assessment method. A new method for detection of delirium. Ann Intern Med. 1990;113:941-948.

26. Glaser BG, Strauss AL. The Discovery of Grounded Theory. Strategies for Qualitative Research. Chicago, IL: Aldine; 1967.

27. Guest G, Bunce A, Johnson L. How many interviews are enough? Field Methods. 2006;18:59-82.

28. Tinetti ME, Baker DI, McAvay G, et al. A multifactorial intervention to reduce the risk of falling among elderly people living in the community. N Engl J Med. 1994;331:821-827.

29. Inouye SK, Bogardus ST, Charpentier PA, et al. A multicomponent intervention to prevent delirium in hospitalized older patients. N Engl J Med. 1999;340:669-676.

30. Brown CJ, Roth DL, Peel C, Allman RM. Predictors of regaining ambulatory ability during hospitalization. J Hosp Med. 2006;1:277-284.

31. Tucker D, Molsberger SC, Clark A. Walking for Wellness: a collaborative program to maintain mobility in hospitalized older adults. Geriatr Nurs. 2004;25:242-245.

32. O'Keefe J, Jack CIA, Lye M. Use of restraints and bed rails in a British hospital. J Am Geriatr Soc. 1996;44:1086-1088.

33. Hangar HC, Ball MC, Wood LA. An analysis of falls in the hospital: can we do without bedrails? J Am Geriatr Soc. 1999;47:529-31.

34. Capezuti E, Maislin G, Strumpf N, Evans LK. Side rail use and bed-related outcomes among nursing home residents. J Am Geriatr Soc. 2002;50:90-96. 